\title{
ESQUISTOSOMIASIS VESICAL: A PROPÓSITO DE UN CASO Y REVISIÓN DE LA LITERATURA
}

Mario Álvarez Maestro, Emilio Ríos González, Purificación Domínguez García', Jorge Vallejo

Herrador, Jesús Díez Rodríguez y Luis Martínez-Piñeiro.

Servicio de Urología y Anatomía Patológica'. Hospital Infanta Sofía. Madrid. España.

Resumen.- OBJETIVO: Aportación de un nuevo caso de esquistosomiasis vesical.

MÉTODOS: Paciente de 21 años que acude a nuestra consulta con hematuria de varias semanas de evolución, observando en la cistoscopia la existencia de lesiones rojizas sobreelevadas que sugieren la existencia de un tumor vesical atípico.

RESULTADOS: Tras ser sometido a un análisis de sangre y orina rigurosos, pruebas de imagen y, posteriormente, resección transuretral vesical el paciente es diagnosticado en el estudio anatomopatológico de esquistosomiasis vesical.

\section{CORRESPONDENCIA}

Mario Álvarez Maestro

Servicio de Urología Hospital Infanta Sofía

Paseo Europa, 34

28703 San Sebastian De Los Reyes Madrid (España)

malvarezmaestro@hotmail.com

Aceptado para publicar: 17 de noviembre 2009 
CONCLUSIONES: Dada la elevada prevalencia de la esquistosomiasis en los países subsaharianos y sus posteriores implicaciones clínicoepidemiológicas, la aparición de una hematuria macro o microscópica en inmigrantes de raza negra, o incluso la aparición de cualquier sintomatología urológica, necesita una evaluación completa, al menos con un estudio parasitológico que considere la bilharziasis como la causa más probable.

Los pacientes que han padecido esquistosomiasis urinaria severa deben someterse a un seguimiento a largo plazo para prevenir y detectar el desarrollo de carcinoma vesical.

Palabras clave: Citología de orina. Hematuria Esquistosoma haematobium.

Summary.- OBJECTIVE: To report a new case of bladder schistosomiasis.

METHODS: A 21 -year-old patient came to our consultation with gross hematuria several weeks before, existing reddish lesions in the cystoscopy that suggested the existence of an atypical bladder tumor.

RESULTS: After intense blood and urine test, ultrasonography and intravenous urography, transurethral resection of bladder was performed and the diagnosis on histological examination was bladder schistosomiasis.

CONCLUSIONS: Given the high prevalence of schistosomiasis in the sub-Saharan countries and later clinical epidemiologic implications, the appearance of macro or microscopic hematuria in black race immigrants, or even any urinary symptoms, it is necessary a complete evaluation, at least with a parasitological analysis (being the bilharziasis the most probably reason).

The patients who have suffered severe urinaryschistosomiasis must complete long-term follow-up to prevent bladder carcinoma.

Keywords: Urine cytology. Hematuria. Schistosoma haematobium.

\section{INTRODUCCIÓN}

Las parasitosis urinarias no son frecuentes en nuestro medio. Según revisión realizada en Pubmed, se han publicado pocos casos en España desde el año 2000. Se trataban en su mayoría de personas provenientes del África Subsahariana, Magreb, Cuenca Mediterránea y Oriente Medio y ocasionalmente en turistas que refieren una estancia previa en países de alta endemia parasitaria $(1,2)$. La presencia de hematuria acompañado, o no de síndrome miccional en un paciente joven originario o viajero reciente en estas zonas debe hacernos pensar en una esquistosomiasis vesical. Ante esta sospecha un método diagnóstico fundamental es la citología urinaria (3).

\section{CASO CLÍNICO}

Presentamos el caso de un varón de 21 años, natural de Gambia (desde hacía 2 años residente en España y con antecedente de malaria), que acude a nuestra consulta por hematuria macroscópica de 3-4 meses de evolución, inicialmente relacionado con infección del tracto urinario por enterobacter cloacae y tratado con fosfomicina por su médico de atención primaria. Ante la persistencia de la hematuria macroscópica intermitente terminal, junto con síntomas irritativos miccionales es remitido a nuestra consulta. La exploración física fue rigurosamente normal, sin alteraciones en los análisis de sangre. En el sedimento de orina se observó > 100 hematíes por campo. Se realizó ecografía donde se observaron lesiones proliferativas intravesicales y engrosamientos nodulares de la pared sugestivas de neoformaciones. En la urografía intravenosa se objetivaron riñones con captación y eliminación adecuada, uréteres y cistograma normal con escaso residuo postmiccional. La cistoscopia flexible confirmó la presencia de varias lesiones sólidas, eritematosas con edema alrededor en fondo, cara anterior y base vesical e incrustaciones blanco-nacaradas de pequeño tamaño, motivo por el cual se sometió a RTU vesical. El estudio anatomopatológico mostró en la submucosa una intensa reacción inflamatoria formada por linfocitos y abundantes eosinófilos. Entre las células inflamatorias había gran cantidad de huevos de Esquistosoma (Figura 1 y 2 ).

El estudio citológico reveló la presencia de huevos de Schistosoma haematobium con su típico espolón o espina terminal, característica de la especie (Figura 3).

Se procedió a tratamiento médico con Praziquantel (Biltricide $囚$ ) a dosis de $40 \mathrm{mg} / \mathrm{Kg}$ en un sólo día en dos tomas. A los 3 meses, se controló al paciente con examen parasitológico en fresco de orina, citología y ecografía vesical no objetivándose persistencia de la enfermedad.

\section{DISCUSIÓN}

\section{Epidemiologia:}

Se calcula que unos 250 millones de personas en las regiones tropicales del planeta padecen esquistosomiasis. Constituye la segunda causa de morbilidad y mortalidad después de la malaria. La esquistosomiasis urinaria es una enfermedad parasitaria endémica producida por Schistosoma haematobium. Este parásito se distribuye ampliamente por el África Subsahariana, costa este africana, Magreb, Chipre y Oriente. 
Medio $(1,2)$. La esquistosomiasis es una enfermedad conocida desde la antigüedad egipcia, como lo demuestran las representaciones de la Estela de Bak y el Papiro de Ebers, $1534 \mathrm{aC}$. donde se describen meticulosamente sus síntomas. Fue en 1851, cuando Theodore Bilharz que trabajaba en un hospital de El Cairo, identificó postmortem las formas adultas de Schistosoma haematobium. Afecta tanto a varones como a mujeres, con una incidencia que alcanza su pico en edades adolescentes, entre 11 y 15 años.

Se reconocen varias especies de esquistosomas: $S$. mansoni, S. japonicum, S. mekongi, S. malayensis, S. haematobium, S. intercalatum y S. mattheii. Unicamente el S. haematobium produce afectación urinaria, las especies restantes causan manifestaciones digestivas (4). La enfermedad se adquiere al bañarse en aguas contaminadas. Los parásitos penetran al ser humano a través de la piel en aguas dulces contaminadas como balsas y pantanos que contengan "cercarias" (formas infestantes) y lo infectan. Una vez que los esquistosomas pasan a la circulación sanguínea, tienen un tropismo especial por los plexos perivesicales (S. haematobium) o perihemorroidales (resto de especies).

\section{Clínica:}

En la evolución clínica de la bilharziasis urinaria se diferencian cuatro fases $(1,5,6)$ :

- Manifestaciones cutáneas locales iniciales: prurito y enrojecimiento a las pocas horas de un baño en agua infectada ("prurito del nadador").

- De cuatro a ocho semanas tras la infección, ocurre la fase de invasión o toxémica, coincidiendo con la prime-

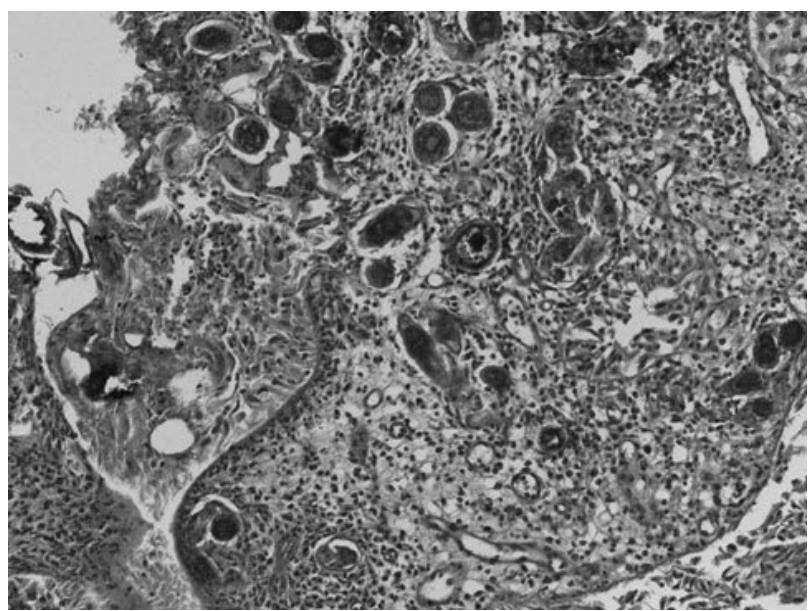

FIGURA 1. Tinción con hematoxilina-eosina donde se observan en la submucosa una intensa reacción inflamatoria formada por linfocitos y abundantes eosinófilos. Entre las células inflamatorias había gran cantidad de huevos de Esquistosoma. ra puesta de huevos. Puede ser asintomática o producir el Síndrome de Katayama con fiebre, urticaria, cefaleas, artralgias, dolor abdominal, hepatoesplenomegalia, linfadenopatías y eosinofilia.

- En un estadio más avanzado, meses o años tras la exposición al parásito aparecen los síntomas genitourinarios correspondientes a la eliminación de los huevos en la orina. Aparece el síntoma fundamental, la hematuria, de tipo terminal, intermitente y recidivante. Se puede acompañar de otros síntomas urinarios como síndrome irritativo inespecífico, polaquiuria, dolor suprapúbico con la micción, etc. El síndrome de "vejiga contraída" esquistosomiásico se observa con mayor frecuencia durante el estadio crónico activo tardío, cuando la carga de huevos es máxima (dolor abdominal inferior profundo constante + dolor pelviano + urgencia + incontinencia). El diagnóstico en esta fase se realiza observando huevos de Schistosoma en la orina (La recogida de orina debe realizarse entre las 11 am y las 2 pm momento en el que, al parecer, la excrección del parásito es máxima. El recuento de la cantidad de huevos en la muestra de orina permite evaluar la severidad de la infección, que se considerará severa cuando sea superior a 400 huevos por cada $100 \mathrm{ml}$ de orina).

Superadas estas fases, tras unos años de infestación aparecen las secuelas:

- La uropatía bilharziana: ésta se produce por la intensa reacción inflamatoria granulomatosa y las lesiones fibrosas irreversibles producidas en respuesta a los huevos del parásito. Las lesiones que aparecen con más frecuencia en este período son la esclerosis- calcificación de la pared vesical y ureteral. La estenosis ureteral es una de las más graves complicaciones y de las que

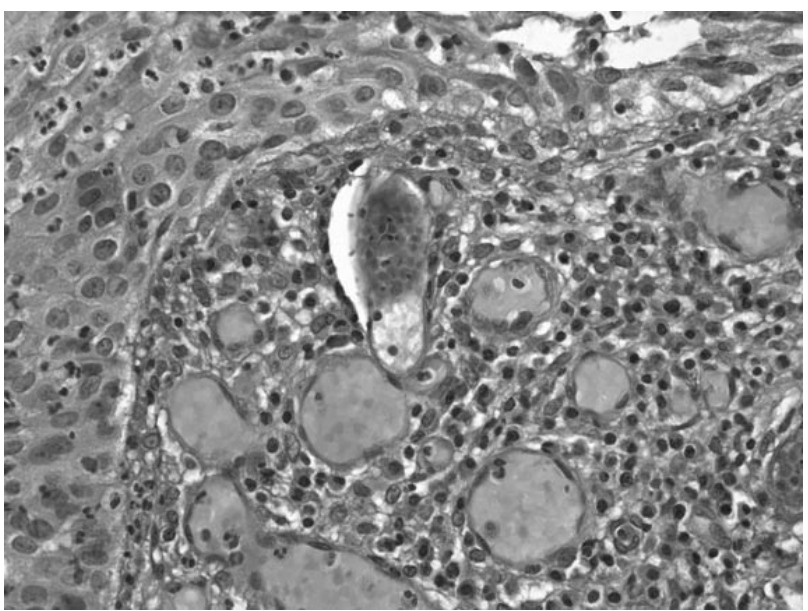

FIGURA 2. Imagen microscópica de vejiga urinaria en la que se observa un infiltrado inflamatorio difuso y focos de calcificación, alrededor de una típica sección transversal de esquistosoma haematobium "in situ". HE x 60. 
más compromete el pronóstico vital. Suele ser asintomática y debutar con una uropatía obstructiva que si es bilateral puede terminar en insuficiencia renal crónica. Afecta generalmente al uréter yuxtavesical, por la localización pelviana del parásito, aunque puede ascender hacia pelvis y ocasionar cólicos nefríticos, pielonefritis o pionefrosis. El carcinoma vesical es otra de las posibles complicaciones, la mayoría de los tumores de la vejiga bilharziásica son carcinomas escamosos. Además, la incidencia de carcinoma de células transicionales también está aumentada, siendo estos más agresivos, de alto grado, con elevada tendencia a la recurrencia y de afectar a personas más jóvenes.

Algunos estudios demuestran que en zonas endémicas el $31 \%$ de los pacientes con cáncer vesical tienen antecedentes de esquistosomiasis vesical, siendo el 60\% carcinomas escamosos y el $5-15 \%$ de otras estirpes.

\section{Etiopatogenia:}

Diversas teorías explican cómo los huevos del parásito liberan unas sustancias que favorecen la acción oncogénica de otros productos carcinógenos como son los metabolitos del triptófano, compuestos $\mathrm{N}$-nitrosos y la betaglucuronidasa.

La afectación del aparato genital femenino es más frecuente que la del masculino, afectando a ovarios, trompas de Falopio, útero y vagina. La esquistosomiasis genital es causa de infertilidad en amplias zonas de Africa. No hay informes documentados de esquistosomiasis fetal.

Los huevos del parásito son depositados lo más cerca posible de la mucosa del tracto urinario, pasando desde las áreas de ulceración granulomatosa de la mucosa hacia la orina, donde pueden identificarse microscópicamente. Durante la infección activa causan una gran irritación en los tejidos. En etapas precoces, el exudado inflamatorio puede contener abundantes eosinófilos e incluso formar granulomas. Los huevos situados más profundamente acaban muriendo y se calcifican, a veces en tal cantidad que se pueden verse en la radiología. Estos huevos calcificados se rodean de un tejido fibroso denso, producto de la reacción inflamatoria crónica, y pueden formar pólipos y masas que llegan a causar obstrucciones urinarias. La severa inflamación crónica puede causar proliferación y cambios metaplásicos en la mucosa, y predispone al desarrollo de enfermedades malignas, sobre todo carcinomas escamosos (7). La mayoría de casos ocurridos en España son importados; los casos "agudos" por turistas de países con alta endemia parasitaria y los casos "crónicos" por los colectivos de inmigrantes africanos; favorecida además la parasitación en este último colectivo por las condiciones de vida de los inmigrantes en nuestro país (situación legal irregular, falta de asistencia sanitaria, desconocimiento del idioma, etc.) que dificultan el diagnóstico y tratamiento en los portadores del trematodo (8).

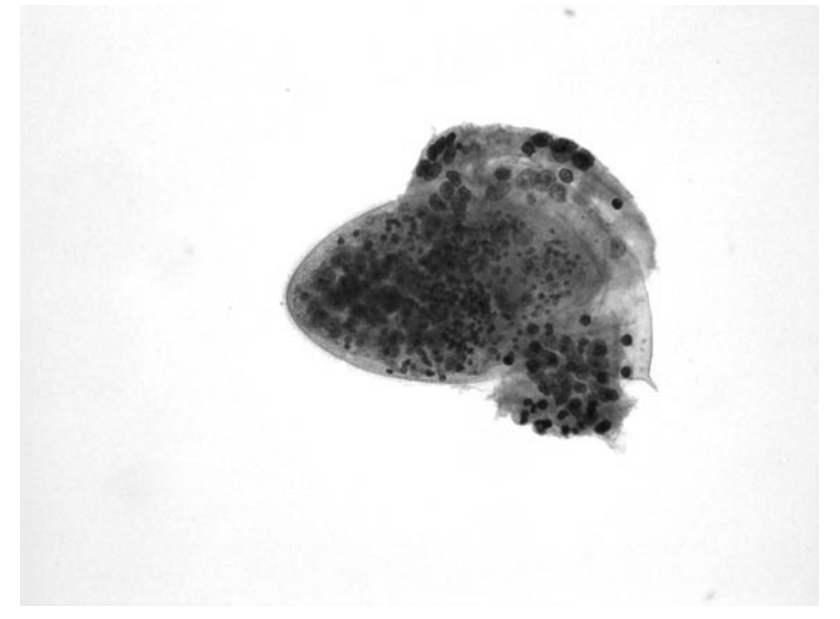

FIGURA 3. Extendido citológico de orina, en el que pueden observarse huevos de esquistosoma haematobium con su típica espina terminal.

\section{Diagnóstico:}

La radiología es la herramienta diagnóstica para la evaluación de secuelas y complicaciones. La clásica presentación de una vejiga calcificada o "vejiga en porcelana", similar a una cabeza fetal, es patognomónica de la esquistosomiasis urinaria crónica (9). En la urografía intravenosa, se observan con facilidad hidrouréter, hidronefrosis, riñón anulado, riñón no funcionante, estenosis ureteral y defectos de llenado vesical y ureteral (lesiones polipoides). La cistografía evidencia la posible disminución de la capacidad vesical o aparición de defectos de repleción que deberán ser evaluados mediante cistoscopia.

Los estudios endoscópicos permiten visualizar directamente las lesiones y obtener biopsias de las mismas. En la primera fase de la enfermedad aparecen granulomas, erosiones y congestión de la mucosa. Las lesiones secundarias son nódulos bilharzianos (mucosa vesical sobreelevada por formaciones papulares con aspecto de grano de arroz). El bilharzioma aparece en la fase terciaria (formación redondeada, pediculada o sesil, de coloración rojiza en las paredes posteriores y laterales de la vejiga). La afectación crónica de la vejiga produce unas lesiones parcheadas, planas, de color parduzco con tendencia a la fibrosis y a la retracción vesical que se llaman "parches arenosos" por su aspecto endoscópico. Una característica importante de la esquistosomiasis vesical es que podemos encontrar lesiones coexistentes en diferentes estadios evolutivos.

El diagnóstico definitivo lo dan la presencia de granulomas y huevos de Esquistosoma en la submucosa de las biopsias vesicales. 


\section{Tratamiento:}

El tratamiento con Praziquantel (Biltricide $\AA$ ) a dosis de $40 \mathrm{mg} / \mathrm{Kg}$ administrado vía oral en una única dosis o $20 \mathrm{mg} / \mathrm{Kg}$ (dos dosis en un día) es la pauta habitual (es activo contra todas las formas y estadios clínicos de la enfermedad). Como alternativa se puede administrar Oxamniquina (sólo efectivo contra S. Mansoni) $(1,10)$. Con ello se suele producir una regresión de la reacción inflamatoria producida por la oviposición, así como la curación de la infección en la gran mayoría de los casos. El éxito del tratamiento se determina al confirmar que los huevos desaparecen de la orina. Las lesiones proliferativas intravesicales deben ser resecadas para información histológica y reducir los síntomas irritativos miccionales así como la hematuria (como en nuestro caso).

El Praziquantel es ineficaz para las lesiones cicatriciales terciarias que deberán ser tratadas mediante los procedimientos quirúrgicos adecuados: reimplante ureteral, resección y anastomosis terminoterminal, dilataciones ureterales endoscópicas, sustitución de uréter por un segmento ileal, cistoplastia de ampliación, cistectomía parcial, incisión endoscópica en las resecciones de cueIlo... hasta nefrectomía en riñones hidronefróticos con pobre función y patología asociada (litiasis, infección). Los viajeros a zonas endémicas deben conocer los peligros de los cursos de agua, ríos, lagunas y lagos infestados, y evitar bañarse o utilizar estas aguas. Las cercarias mueren al hervir el agua. En la actualidad, ningún fármaco preventivo ha demostrado efectividad contra la infección por S. haematobium.

\section{CONCLUSIONES}

- Uno de los propósitos de este trabajo ha sido el de concienciar a los urólogos de nuestro medio de la necesidad de tener presentes enfermedades tropicales que hasta ahora eran muy infrecuentes pero que debido al aumento de inmigrantes que proceden de zonas endémicas, favorecen la posibilidad de que nos encontremos, en la práctica diaria, ante pacientes que pueden tener una enfermedad parasitaria importada.

- En particular, la esquistosomiasis es una de las enfermedades tropicales prioritarias para la Organización Mundial de la Salud.

- Se considera necesario realizar análisis de orina con estudio parasitológico del sedimento urinario a aquellos inmigrantes procedentes del África subsahariana que presentan cualquier síntoma urinario, para detectar numerosos casos de bilharziasis vesical que de lo contrario podrían pasar inadvertidos.

\section{BIBLIOGRAFÍA y LECTURAS RECOMENDADAS ( ${ }^{*}$ lectura de interés $y^{* *}$ lectura fundamental)}

**1. Bichler KH, Savatovsky I; the Members of the Urinary Tract Infection (UTI) Working Group of the Guidelines Office of the European Association of Urology (EAU): Kurt G. Naber (Chairman)*, Michael C. Bischop, Truls E. Bjerklund-Johansen,

Henry Botto, Mete Cek, Magnus Grabe, Bernhard Lobel, Juan Palou Redorta,

Peter Tenke.

*2. Ross AG, Bartley PB, Sleigh AC, Olds GR, Li Y, Williams GM et al. Schistosomiasis. N Engl J Med 2002; 346:1212-20.

*3. Mayayo Artal E, Sánchez Herrera S, Razquin Lizarraga S, Antón Valenti E. Esquistosomiasis (Bilharziasis). Importancia de la citología. A propósito de dos casos. Arch Esp Urol, 1993; 46 (10):907-909.

4. Morales D, Molina J, Martínez Ortiz A, Martínez Artola V, Beristáin X. Hematuria intermitente. Esquistosomiasis vesical. A propósito de un caso. An. Sist. Sanit. Navar. 2009; 32 (2): 265-268

5. Sanz Chinesta S, Pontones Moreno JL, Paya Roma A, Boronat Tormo F, Martínez Sarmiento M, Jiménez Cruz JF. Bilarziosis en vejiga. Arch Esp Urol, 1996; 49(6):545-9.

6. Donate Moreno MJ, Pastor Navarro H, Giménez Bachs JM, Carrión López P, Segura Martín M,Salinas Sánchez AS et al. Esquistosomiasis vesical, aportación de un caso y revisión de la literatura española. Actas Urol Esp, 2006; 30(7):714-9.

7. Navarro Cabañas G, García Sánchez N, Rubio Rubio R, Izaguirre Zugazaga C, Clavel Parrilla A, Seral García C. Esquistosomiasis urogenital: un diagnóstico sencillo. An Esp Pediatr, 2006; 64: 284-2.

8. Borrell Palanca A, Queipo Zaragozá JA, Beltrán Meseguer JF, Pérez C.F., Trullenque E.F., Sempere P.F.. Infección vesical por esquistosoma: una causa inhabitual de hematuria. Actas Urol Esp, 2008; 32(2):253-5.

9. López López AI, Cao Avellaneda E, Prieto González A, Ferri Ñ́guez B, Maluff Torres A, Pérez Albacete M. Esquistosomiasis: una parasitosis urinaria cada vez más frecuente. Actas Urol Esp, 2007; 31(8):9158.

$* * 10$. Rollinson D. A wake up call for urinary schistosomiasis: reconciling research effort with public health importance. Parasitol, 2009; 136(12):1593-610. 